\title{
CINÉTICA DE LIBERAÇÃO DE CHUMBO DE SOLOS DE ÁREA DE MINERAÇÃo E METALURGIA DE METAIS PESADOS ${ }^{(1)}$
}

\author{
Brenda Novaes Buschle ${ }^{(2)}$, Jussara Kasuko Palmeiro ${ }^{(2)}$, Youssef Bacila \\ Sade $^{(2)}$, Vander de Freitas Melo ${ }^{(3)}$, Maurício Gomes de Andrade ${ }^{(4)}$ \& Araína \\ Hulmann Batista ${ }^{(5)}$
}

\begin{abstract}
RESUMO
O estudo de cinética de liberação de metais pesados é uma importante ferramenta de diagnóstico ambiental de áreas contaminadas, pois determina, além dos teores acumulados liberados após tempos crescentes de equilíbrio, a taxa (velocidade) de dessorção desses poluentes para a solução do solo. Com o objetivo de avaliar a cinética de liberação de $\mathrm{Pb}$ de solos da área de mineração e metalurgia de metais pesados, no município de Adrianópolis (PR), vale do rio Ribeira, selecionaram-se oito solos, submetidos a diferentes formas de contaminação (solos 2, 4 e 5 - incorporação de resíduos da metalurgia de metais pesados aos perfis; solos 3, 6 e 7 - adição de $\mathrm{Pb}$ particulado via chaminés da fábrica; solo 8 - contaminação por passagem da água pluvial pela fábrica desativada, que escorre em direção ao rio Ribeira). O solo 1 , sob mata nativa, fora da direção de caminhamento das fumaças da metalurgia, localizado a $1.560 \mathrm{~m}$ de distância e a $380 \mathrm{~m}$ acima da cota da fábrica desativada, foi escolhido como referência dos teores naturais de $\mathrm{Pb}$ dos solos da região. As amostras de solo, coletadas em duas profundidades (0 a 10 e 20 a $40 \mathrm{~cm}$ ), foram submetidas a extrações sequenciais com ácido cítrico $0,1 \mathrm{~mol} \mathrm{~L}^{-1}$ após diferentes períodos de contato (tempos acumulados: $2,14,38,86,182,326,518$, 806 e $1.382 \mathrm{~h}$ ). Os teores totais de $\mathrm{Pb}$ (extração com $\mathrm{HF}$ e $\mathrm{HNO}_{3}$ concentrados e $\mathrm{H}_{2} \mathrm{O}_{2} 30 \% \mathrm{v} / \mathrm{v}$ ) foram altos (máximo de $24.755,6 \mathrm{mg} \mathrm{kg}^{-1}$ ) e indicaram intensa contaminação dos solos. Os dados da cinética de liberação foram ajustados às equações parabólicas de difusão, sendo a liberação do $\mathrm{Pb}$ gradual e bifásica, com velocidade de liberação maior na primeira fase. Os solos 3 e 5 foram considerados com elevado potencial de contaminação ambiental devido aos altos teores de $\mathrm{Pb}$ dessorvido acumulados nas extrações sequenciais com ácido cítrico (máximo de
\end{abstract}

\footnotetext{
(1) Parte do trabalho de conclusão de curso de graduação dos três primeiros autores, apresentado à Universidade Tecnológica Federal do Paraná - UTFPR. Recebido para publicação em novembro de 2009 e aprovado em setembro de 2010.

(2) Tecnólogo em Química Ambiental. Universidade Tecnológica Federal do Paraná - UTFPR. Av. Sete de Setembro 3165, CEP 80203-901 Curitiba (PR). E-mails: brendabuschle@yahoo.com.br; jussarakp@ig.com.br; yousade@ufpr.br

(3) Departamento de Solos e Engenharia Agrícola, Universidade Federal do Paraná - UFPR. Rua dos Funcionários 1540, Juvevê, CEP 80035-050 Curitiba (PR). Bolsista CNPq. E-mail: vanderfm@ufpr.br

(4) Departamento de Química e Biologia, UTFPR. E-mail: mandrade@utfpr.edu.br

(5) Engenheira-Agrônoma, Mestranda em Ciência do Solo, UFPR. E-mail: arainahb_solos@hotmail.com
} 
$\left.18.577,4 \mathrm{mg} \mathrm{kg}^{-1}\right)$. O solo 6, com baixos teores de argila, apresentou a maior velocidade (taxa) de dessorção de $\mathrm{Pb}$.

Termos de indexação: adsorção específica de $\mathrm{Pb}$, equação parabólica de difusão, ácido cítrico.

\title{
SUMMARY: KINETICS OF LEAD RELEASE FROM SOILS IN HEAVY METAL MINING AND METALLURGY AREA
}

\begin{abstract}
Kinetics studies of heavy metal release are an important form of environmental diagnostics in contaminated areas, due to the possibility of determining, aside from the total release contents after increasing equilibrium times, the rate (velocity) of heavy metal to soil solution. The objective of this study was to determined $\mathrm{Pb}$ kinetics release from soils of the heavy metal mining and metallurgy area in Adrianópolis (PR), Brazil, in the Ribeira river valley. Eight soils were exposed to different contamination forms: (soils 2, 4 and 5 -residue incorporated in the soil profile; soils 3, 6 and 7 - sprinkled with particulate matter from the factory chimney; soil 8 - contamination by rain water flowing from the shut down factory towards the Ribeira river). Soil 1, under native forest, was chosen as reference of the natural soil Pb levels. The soil samples, collected from two layers $(0-10 \mathrm{~cm}, 20-40 \mathrm{~cm})$, were subjected to sequential extractions with $0.1 \mathrm{~mol} \mathrm{~L}^{-1}$ citric acid during different periods (2; 14; 38; 86; 182; 326; 518; 806; and 1,382 h). The high total $\mathrm{Pb}$ contents (extracted by concentrated $\mathrm{HF}_{\text {and }} \mathrm{HNO}_{3}$ and $30 \%(\mathrm{v} / \mathrm{v})$ $\mathrm{H}_{2} \mathrm{O}_{2}$ ) (maximum content of 24,755.6 $\mathrm{mg} \mathrm{kg}^{-1}$ ) revealed intense soil contamination. The release kinetics data were fitted to parabolic equations of diffusion; lead release was gradual and biphasic (higher in the first phase). The environmental risks were considered high in the soils 3 and 5, where the evaluated levels of total Pb accumulated were high (reaching $18,577.4 \mathrm{mg} \mathrm{kg}^{-1}$ ). The $P b$ release rate was highest from soil 6 , where clay content was low.
\end{abstract}

Index terms: Pb specific adsorption; parabolic diffusion equation; citric acid.

\section{INTRODUÇÃO}

As atividades de mineração são importantes geradoras de passivos ambientais. Os principais resíduos sólidos gerados por atividades mineradoras são o estéril (porção de rocha ou solo que recobre a fração que contém o metal desejado) e os rejeitos (decorrentes do beneficiamento do minério). Esses dois resíduos sólidos são geralmente depositados em áreas de grande extensão, inviabilizando uma gama de atividades e servindo como fonte de dispersão da poluição (Amaral Sobrinho et al., 1998; Dias-Júnior et al., 1998; Bosso et al., 2008).

No município de Adrianópolis (PR), vale do rio Ribeira, foram desenvolvidas por quase 50 anos as atividades de mineração e metalurgia de primeira fusão de metais pesados, principalmente $\mathrm{Pb}$ (mineral galena - PbS). Em 1995, a mineradora encerrou suas atividades, deixando a céu aberto cerca de $177 \mathrm{Gg}$ de resíduos de beneficiamento (Eysink, 1988; Cassiano, 2001) à disposição das chuvas, enxurradas e ventos e ao contato direto da população, da flora e da fauna. Outros estudos de diagnóstico e de remediação ambiental foram desenvolvidos na área: Kummer (2008) determinou as características físico-químicas e mineralógicas dos solos e promoveu a especiação de $\mathrm{Pb}$ e $\mathrm{Zn}$ por meio de extrações seqüenciais; nos trabalhos de Barros (2010a, b) foram gerados indicadores de qualidade dos solos por meio de avaliações físicas e químicas, microbiológicas, da mesofauna e de plantas de ocorrência natural na área; Andrade (2009) encontrou teores de $\mathrm{Pb}$ nas águas coletadas nas redes de drenagens da área acima do limite permitido pela Resolução Conama 357/05, e os teores de $\mathrm{Pb}$ nos sedimentos atingiram $24.300 \mathrm{mg} \mathrm{kg}^{-1}$; e Andrade et al. (2009a,b), em estudos de fitoextração e de disponibilidade de metais pesados, concluíram que o girassol foi a espécie com maior potencial para a remediação dos solos com nível intermediário de contaminação e a extração com $\mathrm{HNO}_{3} \quad 0,5 \mathrm{~mol} \mathrm{~L}^{-1}$ fervente deve ser preferida para predição da biodisponibilidade de $\mathrm{Pb}$ e $\mathrm{Zn}$ nos solos da área.

Teores totais elevados, associados com taxa de dessorção rápida de metais pesados para a solução do solo, são condições mais deletérias ao meio ambiente, por facilitar a entrada desses poluentes na cadeia trófica e contaminar as águas subterrâneas (Roca \& Pomares, 1991). Existe um equilíbrio entre as formas de metais no solo: as plantas absorvem o $\mathrm{Pb}$ da solução, o qual é tamponado pelas formas trocáveis, que são normalmente repostas pelas reservas do solo (formas não trocáveis e estruturais) (Alloway, 1990).

Os métodos de extração que simulam a dinâmica de absorção de $\mathrm{Pb}$ pelas plantas descrevem mais 
apropriadamente a cinética de liberação desse metal em condições de campo. Um coquetel de ácidos orgânicos de baixa massa molar (ácidos acético, fórmico, cítrico e málico, ajustados ao pH do solo) foi utilizado na comparação entre metal pesado extraído do solo e fração biodisponível para o milho (Wang et al., 2003). Na região da rizosfera, em relação à matriz do solo, são encontradas maiores concentrações de compostos orgânicos, como: aminoácidos, açúcares, ácidos orgânicos, lipídios, proteínas e enzimas, em virtude da exsudação das raízes e intensa atividade microbiana (Ali et al., 2000). Esses compostos estão diretamente relacionados com os processos de acidificação, complexação, precipitação e oxirredução que ocorrem na rizosfera, influenciando a liberação de formas não trocáveis e estruturais de metais pesados para as plantas e organismos do solo (McGrath \& Zahao, 2003; Xu et al., 2007). Os ácidos orgânicos de baixa massa molar, como oxálico, cítrico, lático, succínico, entre outros, estão envolvidos nos processos de intemperismo dos minerais por meio da formação de complexos ácidos orgânicos-metal, trocas de ligantes e reações de protonação (Simard et al., 1992).

A cinética de dessorção sob diferentes condições experimentais visa simular as condições naturais de liberação de metais no solo (Melo et al., 2005). Simard et al. (1992), trabalhando com diferentes solos do Canadá, tempos de extração (equilíbrio) com ácido cítrico e distintas equações de cinética de liberação (Elovich, equação parabólica de difusão, equação função potência, equação de primeira ordem e equação de segunda ordem), verificaram que a equação parabólica de difusão descreveu, com maior exatidão (maior coeficiente de determinação e menor erro-padrão estimado), a cinética de liberação de $\mathrm{K}$ e $\mathrm{Mg}$ nas condições do estudo. As equações de difusão parabólica e de primeira ordem foram adequadas para descrição da cinética de liberação de $\mathrm{Pb}$ de solos argilosos e arenosos do Egito, sob duas condições de temperatura (Elkhatib et al., 1992).

O objetivo deste trabalho foi avaliar a concentração e a taxa de liberação de $\mathrm{Pb}$ de solos da área de mineração e metalurgia de metais pesados, no município de Adrianópolis (PR), vale do rio Ribeira, por meio de extrações sucessivas com ácido cítrico $0,1 \mathrm{~mol} \mathrm{~L}^{-1}$.

\section{MATERIAL E MÉTODOS}

\section{Área de estudo e amostragem dos solos}

Aárea situa-se no município de Adrianópolis, região metropolitana de Curitiba, Paraná, mais precisamente na localidade de Panelas de Brejaúvas, distante $12 \mathrm{~km}$ da sede do município, próxima às margens do rio Ribeira, fronteira com o Estado de São Paulo.

$\mathrm{O}$ estudo foi desenvolvido a partir de oito perfis de solos de diferentes locais dentro da antiga área de mineração e metalurgia de chumbo (Figura 1 e
Quadro 1). Tomou-se o solo 1 como referência dos teores naturais de $\mathrm{Pb}$ nos solos, por estar sob vegetação nativa e mais distante $(1.560 \mathrm{~m})$ e com maior diferença de cota $(380 \mathrm{~m})$ das atividades industriais (antiga fábrica). Outros autores fizeram a mesma escolha (Kummer, 2008; Andrade et al., 2009a,b; Barros et al., 2010a,b). Foram coletadas amostras nas camadas de 0-10 e 20-40 cm de profundidade. Os critérios para escolha dos locais de amostragem, levando em consideração principalmente os teores de $\mathrm{Pb}$ e a modalidade de contaminação (resíduos sólidos do beneficiamento e particulados oriundos das chaminés) da área, são apresentados por Kummer (2008) e Andrade et al. (2009a,b).

Após extração com $\mathrm{HF}$ e $\mathrm{HNO}_{3}$ concentrados e $\mathrm{H}_{2} \mathrm{O}_{2} 30 \%$ (v/v) em forno de micro-ondas (potência de $1.000 \mathrm{~W}$, temperatura de $175 \pm 5^{\circ} \mathrm{C}$, por $\left.10 \mathrm{~min}\right)(\mathrm{Lim}$ \& Jackson, 1986), os teores totais de $\mathrm{Pb}$ foram determinados por espectroscopia de emissão atômica com plasma acoplado indutivamente (ICP-AES) (Quadro 2).

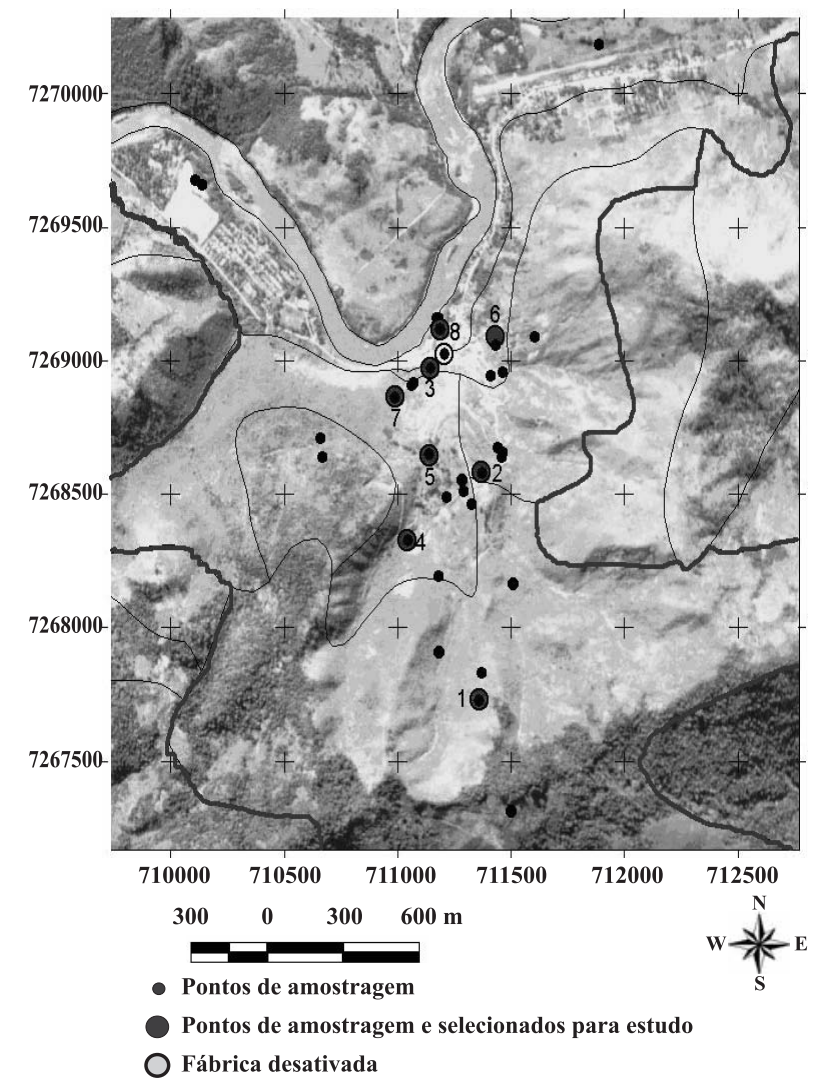

Base cartográfica: Carta de Apiaí, IBGE, Secretaria de Planejamento da Presidência da República.

Sistema de Projeção: Universal Transversa de Mercator. Datum: SAD-69.

Figura 1. Foto aérea georreferenciada com os compartimentos geomorfológicos (linhas finas), localização da fábrica desativada, pontos de amostragem para reconhecimento da área (círculos pretos pequenos) e locais selecionados para o presente estudo (solos 1 a 8 - círculos pretos maiores). 
Quadro 1. Localização, classificação dos solos e características dos locais de amostragem identificadas em campo

\begin{tabular}{|c|c|c|c|c|c|}
\hline \multirow[b]{2}{*}{ Solo } & \multicolumn{3}{|c|}{ UTM (22J) } & \multirow{2}{*}{\multicolumn{2}{|c|}{ Observações }} \\
\hline & $\begin{array}{c}\mathrm{N}-\mathrm{S} \\
\text { Latitude }\end{array}$ & $\begin{array}{c}\text { E-W } \\
\text { Longitude }\end{array}$ & $\begin{array}{c}\text { Altitude/ } \\
\text { Distância (1) }\end{array}$ & & \\
\hline 1 & $7267313 \mathrm{~S}$ & $711502 \mathrm{~W}$ & $546 / 1.560$ & Neossolo Litólico & $\begin{array}{l}\text { Solo de referência sob mata nativa. Graças à grande distância (1.700 } \\
\text { m) e altitude do local em relação à fábrica (diferença de cota de } 380 \\
\text { m), não havia evidências da influência direta das atividades de } \\
\text { mineração no solo. Material de origem: rochas } \\
\text { carbonáticas/complexos granitoides. }\end{array}$ \\
\hline 2 & 7268164 & 711513 & $326 / 563$ & $\begin{array}{l}\text { Cambissolo } \\
\text { Háplico }\end{array}$ & $\begin{array}{l}\text { Posição intermediária da encosta, com evidência de deposição de } \\
\text { colúvio mais rejeitos da metalurgia vindos das partes mais altas. } \\
\text { Material de origem: rochas carbonáticas/complexos granitóides. }\end{array}$ \\
\hline 3 & 7268555 & 711287 & $165 / 45$ & Neossolo Litólico & $\begin{array}{l}\text { Próximo à fábrica (Figura 1), com os horizontes do solo preservados e } \\
\text { sem incorporação de rejeitos sólidos da metalurgia. Perfil na base da } \\
\text { encosta. Solo com potencial (muito próximo da fábrica e localizado } \\
\text { no sentido de caminhamento dos ventos) de adição de } \mathrm{Pb} \\
\text { particulado, por meio da emissão de fumaça pelas chaminés. Material } \\
\text { de origem: rochas carbonáticas/complexos granitoides. }\end{array}$ \\
\hline 4 & 7267907 & 711187 & $327 / 590$ & $\begin{array}{l}\text { Mistura de } \\
\text { sedimento mais } \\
\text { rejeito fino }(2)\end{array}$ & $\begin{array}{l}\text { Vale soterrado com pilhas de rejeitos da metalurgia, misturados ao } \\
\text { solo. Como resultado, a camada de coleta de amostra não } \\
\text { apresentava estrutura e horizontes pedogenéticos. Material de } \\
\text { origem: mistura de sedimentos mais rejeitos da metalurgia. }\end{array}$ \\
\hline 5 & 7268070 & 711360 & $316 / 455$ & $\begin{array}{l}\text { Mistura de solo } \\
\text { mais rejeito } \\
\text { grosseiro }(2)\end{array}$ & $\begin{array}{l}\text { Grande ocorrência de rejeitos da metalurgia na superfície e parte } \\
\text { incorporada ao solo. Como resultado, a camada de coleta de amostra } \\
\text { não apresentava estrutura e horizontes pedogenéticos. Material de } \\
\text { origem: mistura de solo mais rejeitos da metalurgia. }\end{array}$ \\
\hline 6 & 7268671 & 711572 & $202 / 295$ & $\begin{array}{l}\text { Neossolo } \\
\text { Quartzarênico }\end{array}$ & $\begin{array}{l}\text { Idem solo } 3 \text {, exceto pela maior distância em relação a fábrica e pelo } \\
\text { material de origem, que é quartzito. }\end{array}$ \\
\hline 7 & 7268499 & 711158 & $194 / 321$ & Cambissolo Háplico & Idem solo 3 , exceto pela maior distância em relação à fábrica. \\
\hline 8 & 7268701 & 711331 & $157 / 64$ & Neossolo Flúvico & $\begin{array}{l}\text { Faixa entre a fábrica e o rio Ribeira e relevo praticamente plano. } \\
\text { Solo com potencial de contaminação pela passagem da água pluvial } \\
\text { pela fábrica, que escorre em direção ao rio Ribeira. Material de } \\
\text { origem: sedimentos arenosos (fluviais). }\end{array}$ \\
\hline
\end{tabular}

(1) Distância em linha reta do ponto de amostragem à fábrica desativada. ${ }^{(2)}$ Resíduo de mineração e metalurgia de chumbo intimamente misturados ao solo; devido à falta de horizontes pedogenéticos, não foi possível classificar os perfis de solos.

Quadro 2. Teores totais de $\mathrm{Pb}$ e resultados de algumas análises químicas e textural da TFSA e mineralógicas da fração argila das amostras dos solos ${ }^{(1)}$

\begin{tabular}{|c|c|c|c|c|c|c|c|c|c|c|c|c|c|}
\hline Amostra & Pb total & $\mathrm{pH} \mathrm{H}_{2} \mathrm{O}$ & CTC pH 7,0 & $\mathrm{CO}$ & Areia & Argila & $\mathbf{C t}$ & Gb & $\mathrm{Hm}$ & Gt & Am & ES & EHE \\
\hline & $\mathrm{mg} \mathrm{kg}^{-1}$ & & $\mathrm{cmol}_{\mathrm{c}} \mathrm{dm}^{-3}$ & $\mathrm{~g} \mathrm{~kg}^{-1}$ & & & - & & & & & & \\
\hline $1(0-10 \mathrm{~cm})$ & 465,1 & 6,7 & 31,7 & 45,0 & 162 & 477 & 537 & 36 & 20 & 8 & 56 & $\mathrm{nq}$ & $\mathrm{nq}$ \\
\hline $1(20-40 \mathrm{~cm})$ & 53,8 & 6,8 & 31,2 & 26,9 & 170 & 516 & 534 & 33 & 19 & 4 & 50 & $\mathrm{nq}$ & $\mathrm{nq}$ \\
\hline $2(0-10 \mathrm{~cm})$ & $4.133,3$ & 6,6 & 34,7 & 37,7 & 98 & 441 & 439 & 51 & 10 & 21 & 58 & $\mathrm{nq}$ & $\mathrm{nq}$ \\
\hline $2(20-40 \mathrm{~cm})$ & $4.007,5$ & 7,7 & 30,5 & 19,0 & 89 & 440 & 442 & 40 & 11 & 32 & 45 & $\mathrm{nq}$ & $\mathrm{nq}$ \\
\hline $3(0-10 \mathrm{~cm})$ & $16.212,3$ & 6,9 & 29,2 & 45,6 & 204 & 434 & 534 & 44 & 14 & 12 & 54 & $\mathrm{nq}$ & - \\
\hline $3(20-40 \mathrm{~cm})$ & $19.186,7$ & 6,8 & 28,9 & 37,7 & 188 & 424 & 519 & 41 & 22 & 18 & 26 & $\mathrm{nq}$ & - \\
\hline $4(0-10 \mathrm{~cm})$ & $3.802,7$ & 8,4 & 25,5 & 26,3 & 179 & 266 & 495 & 40 & 0 & 37 & 61 & $\mathrm{nq}$ & $\mathrm{nq}$ \\
\hline $4(20-40 \mathrm{~cm})$ & $7.606,3$ & 8,4 & 31,3 & 6,3 & 99 & 255 & 498 & 40 & 3 & 42 & 35 & $\mathrm{nq}$ & - \\
\hline $5(0-10 \mathrm{~cm})$ & $24.755,6$ & 8,3 & 11,3 & 28,1 & 807 & 48 & 443 & 35 & 3 & 38 & 97 & $\mathrm{nq}$ & - \\
\hline $5(20-40 \mathrm{~cm})$ & $18.192,8$ & 8,0 & 30,5 & 13,0 & 184 & 313 & 445 & 46 & 5 & 27 & 20 & $\mathrm{nq}$ & - \\
\hline $6(0-10 \mathrm{~cm})$ & 802,9 & 4,9 & 7,5 & 11,8 & 730 & 124 & 526 & 21 & 0 & 41 & 58 & - & - \\
\hline $6(20-40 \mathrm{~cm})$ & 48,7 & 4,6 & 3,5 & 6,3 & 777 & 64 & 494 & 24 & 0 & 41 & 75 & - & - \\
\hline $7(0-10 \mathrm{~cm})$ & $3.811,3$ & 8,3 & 27,1 & 30,5 & 151 & 483 & 595 & 35 & 19 & 22 & 69 & $\mathrm{nq}$ & - \\
\hline $7(20-40 \mathrm{~cm})$ & $1.877,5$ & 8,2 & 30,7 & 18,4 & 117 & 549 & 675 & 46 & 11 & 27 & 61 & $\mathrm{nq}$ & - \\
\hline $8(0-10 \mathrm{~cm})$ & $10.141,1$ & 8,3 & 13,3 & 20,8 & 586 & 149 & 502 & 32 & 0 & 39 & 72 & $\mathrm{nq}$ & $\mathrm{nq}$ \\
\hline $8(20-40 \mathrm{~cm})$ & $3.913,7$ & 8,2 & 21,9 & 17,8 & 353 & 318 & 538 & 22 & 8 & 35 & 80 & $\mathrm{nq}$ & $\mathrm{nq}$ \\
\hline
\end{tabular}

(1) CO: carbono orgânico, Ct: caulinita, Gb: gibbsita, Hm: hematita, Gt: goethita, Material amorfo (Am.): extração com oxalato de amônio 0,2 mol L-1, ES: esmectita, EHE: esmectita com hidroxi-Al entrecamadas. nq: mineral apenas identificado por DRX (análise qualitativa).: ausência de reflexão por DRX. Pb total extraído com $\mathrm{HF}$ e $\mathrm{HNO}_{3}$ concentrados e $\mathrm{H}_{2} \mathrm{O}_{2} 30$ \% (v/v). As metodologias para os demais parâmetros podem ser obtidas em Kummer (2008). 
A textura e características químicas da terra fina seca ao ar (TFSA) e mineralogia da argila das amostras são apresentadas no quadro 2, e os detalhes metodológicos podem ser obtidos em Kummer (2008).

\section{Cinética de liberação de $\mathbf{P b}$}

A cinética de liberação de $\mathrm{Pb}$ foi realizada pelo método de extrações sucessivas com ácido cítrico $0,1 \mathrm{~mol} \mathrm{~L}^{-1}$, pH 2,5 (Melo et al., 2005). Em tubos de centrífuga de $100 \mathrm{~mL}$, foram colocados $2,0 \mathrm{~g}$ de TFSA e $20 \mathrm{~mL}$ de ácido cítrico $0,1 \mathrm{~mol} \mathrm{~L}^{-1}$. Após cada tempo de extração, a suspensão foi centrifugada para determinação dos teores de $\mathrm{Pb}$ no sobrenadante por ICP-AES. Âs amostras remanescentes nos tubos de centrífuga adicionaram-se novas alíquotas de $20 \mathrm{~mL}$ do mesmo ácido, repetindo-se esse procedimento até um total de nove extrações. A cada reposição da solução extratora, os tubos foram agitados por uma hora em agitador mecânico horizontal, com cerca de 150 oscilações por minuto. O tempo de contato (agitação mais repouso) de cada extração foi variável $(2,12,24$, $48,96,144,192,288$ e 576 h), resultando no tempo acumulado de $1.382 \mathrm{~h}$ (somatório dos nove tempos individuais de contato solo/solução). Para os tempos de 2,12 e $24 \mathrm{~h}$, os tubos foram agitados por $1 \mathrm{~h}$ e, em seguida, permaneceram em repouso por 1,11 e $23 \mathrm{~h}$, respectivamente. A partir de $48 \mathrm{~h}$ de contato (inclusive), os tubos foram agitados por $1 \mathrm{~h}$ no momento da adição da solução e, a cada $23 \mathrm{~h}$ de repouso, foram novamente agitados por mais $1 \mathrm{~h}$. Por exemplo, para o tempo de $96 \mathrm{~h}$ de extração procedeuse ao seguinte método: $1 \mathrm{~h}$ agitação $+23 \mathrm{~h}$ repouso + $1 \mathrm{~h}$ agitação $+23 \mathrm{~h}$ repouso $+1 \mathrm{~h}$ agitação $+23 \mathrm{~h}$ repouso $+1 \mathrm{~h}$ agitação $+23 \mathrm{~h}$ repouso ( $4 \mathrm{~h}$ de agitação + $92 \mathrm{~h}$ de repouso). O maior tempo individual de contato (576 h) correspondeu a 24 agitações de $1 \mathrm{~h}$ cada, mais 24 procedimentos de repouso de $23 \mathrm{~h}$ cada $(552 \mathrm{~h}$ de repouso). O tempo acumulado de extração de aproximadamente dois meses ( $1.382 \mathrm{~h}$ ) foi descrito em outros estudos como período satisfatório para análise da cinética de liberação de metais em solos (Melo et al., 2005; Elkhatib et al., 1992). A renovação da solução de ácido cítrico nos diferentes tempos de contato objetivou evitar a saturação da solução extratora e estimular a liberação do metal dos constituintes orgânicos e minerais dos solos. Outros autores também adotaram a renovação das soluções extratoras em estudos de cinética de liberação de $\mathrm{Pb}$ (Elkhatib et al., 1992) e de K e Mg (Song \& Huang, 1988; Hundal \& Pasricha, 1993; Hundal \& Pasricha, 1998; Castilhos \& Meurer, 2001; Meurer \& Castilhos, 2001; Melo et al., 2005).

Foram ajustadas aos dados equações parabólicas de difusão: $\mathrm{Mt} / \mathrm{Mo}=\mathrm{a}+\mathrm{kt}^{1 / 2}$ (Hundal \& Pasricha, 1993; Castilhos \& Meurer, 2001), sendo t os tempos de equilíbrios acumulados $(2,14,38,86,182,326$, 518,806 e $1.382 \mathrm{~h}$ ); Mt, os teores acumulados de $\mathrm{Pb}$ até o tempo t; e Mo, os teores acumulados do metal em todo o experimento (somatório dos teores das nove extrações). Os coeficientes angulares (valor k) mediram a taxa de liberação de $\mathrm{Pb}$; quanto maior a inclinação da reta, maior a velocidade de liberação do metal (Elkhatib et al., 1992). O grau de ajuste dos dados à equação parabólica de difusão foi avaliado pelo valor do coeficiente de determinação $\left(\mathrm{R}^{2}\right.$ acima de 0,9$)$ e pelo Erro-Padrão Estimado (baixo EPE) do modelo (Simard et al., 1992; Hundal \& Pasricha, 1993; Hundal \& Pasricha, 1998; Melo et al., 2005) (EPE = $\left[\Sigma\left(\mathrm{M}_{\mathrm{t}}-\mathrm{M}_{\mathrm{t}}{ }^{*}\right)^{2}\right] /(\mathrm{n}-2)$, em que Mt e Mt* representam os teores de $\mathrm{Pb}$ obtidos nas extrações e calculados nas equações de cinética (valores observados e estimados), respectivamente, para o tempo t, e n é o número de pontos).

\section{RESULTADOS E DISCUSSÃO}

O perfil de liberação de $\mathrm{Pb}$ dos solos contaminados (maiores teores totais de $\mathrm{Pb}$ nos solos 2 a 8 em relação ao solo 1 - Quadro 2) com soluções de ácido cítrico (Quadros 3 e 4) apresentou grande variação em função da forma de contaminação do ambiente, identificada em campo no estudo dos perfis e indicada pelas distâncias e posições dos pontos de coletas em relação à fábrica desativada (ver detalhes no Quadro $1 \mathrm{e}$ Figura 1): solos 2, 4 e 5 - incorporação de resíduos da metalurgia ao perfil (Figura 2); solos 3, 6 e 7 - adição de $\mathrm{Pb}$ particulado via chaminés da fábrica (Figura 3); e solo 8 - contaminação por passagem da água pluvial pela fábrica, que escorre em direção ao rio Ribeira (Figura 4). As curvas de dessorção de $\mathrm{Pb}$ da amostra de referência 1 são apresentadas na figura 5 .

A dessorção acumulada de $\mathrm{Pb}$ pela solução diluída de ácido cítrico (tempo de contato acumulado de $1.382 \mathrm{~h}$ ) variou de 26,1 a $18.577,4 \mathrm{mg} \mathrm{kg}^{-1}$ (Quadro 3). Esses valores foram ligeiramente inferiores aos teores totais (extração fluorídrica/nítrica) (Quadro 2); para as amostras de solos contaminados (2 a 8) a proporção de recuperação de $\mathrm{Pb}$ no estudo de cinética $(\mathrm{Pb}$ dessorvido acumulado em $1.382 \mathrm{~h}$ ), em relação aos teores totais, variou de 71 a $96 \%$, valores superiores aos da amostra de referência (amostra 1 - 48 e 59 \%). De acordo com Chlopecka et al. (1996), os metais pesados dos solos vindos do próprio material de origem são menos solúveis que aqueles incorporados por atividades antrópicas. De maneira geral, a dessorção de $\mathrm{Pb}$ com ácido cítrico foi gradual, necessitando de três extrações para liberação de mais de $50 \%$ do acumulado nas nove etapas (Quadro 3). Já na amostra coletada na profundidade de 0 a $10 \mathrm{~cm}$ do solo de referência, foram necessárias cinco etapas de extrações para se atingir essa mesma proporção, indicando maior estabilidade do metal.

Os maiores teores de $\mathrm{Pb}$ dessorvidos em $1.382 \mathrm{~h}$ na camada de 0 a $10 \mathrm{~cm}$ nos solos $1,2,5,6,7$ e 8 (Quadro 3) estão relacionados aos maiores teores totais na superfície e ao maior acúmulo de matéria orgânica (Quadro 2), que previne a lixiviação do metal para a subsuperfície (Kabata-Pendias \& Pendias, 2001). A 
Quadro 3. Percentagem acumulada de $\mathrm{Pb}$ dessorvido nos diferentes tempos de extrações em relação ao teor acumulado em $1.382 \mathrm{~h}$ de contato solo:solução de ácido cítrico $0,1 \mathrm{~mol} \mathrm{~L}^{-1}$ de amostras de solo coletadas em diferentes pontos de antiga área de mineração, no município de Adrianópolis-PR

\begin{tabular}{|c|c|c|c|c|c|c|c|c|c|c|}
\hline \multirow{2}{*}{$\operatorname{Amostra}^{(1)}$} & \multicolumn{9}{|c|}{ Tempo acumulado (h) } & \multirow{2}{*}{$\begin{array}{c}\text { Teor dessorvido } \\
\text { acumulado } \\
\text { em } 1.382 \mathrm{~h}\end{array}$} \\
\hline & 2 & 14 & 38 & 86 & 182 & 326 & 518 & 806 & 1.382 & \\
\hline & \multicolumn{9}{|c|}{$\%^{(2)}$} & $\mathrm{mg} \mathrm{kg}^{-1}$ \\
\hline $1(0-10 \mathrm{~cm})$ & 1,6 & 18,2 & 30,2 & 43,0 & 51,7 & 69,2 & 82,0 & 89,7 & 100,0 & 275,6 \\
\hline $1(20-40 \mathrm{~cm})$ & 70,7 & 76,8 & 77,8 & 89,4 & 89,4 & 89,4 & 97,2 & 99,0 & 100,0 & 26,1 \\
\hline $2(0-10 \mathrm{~cm})$ & 14,2 & 28,1 & 39,2 & 53,8 & 66,6 & 77,3 & 86,7 & 93,9 & 100,0 & $3.669,3$ \\
\hline $2(20-40 \mathrm{~cm})$ & 17,0 & 30,7 & 44,7 & 59,1 & 71,5 & 81,2 & 89,3 & 95,2 & 100,0 & $3.493,8$ \\
\hline $3(0-10 \mathrm{~cm})$ & 17,0 & 30,8 & 42,8 & 56,3 & 68,1 & 78,0 & 86,8 & 94,0 & 100,0 & $15.545,0$ \\
\hline $3(20-40 \mathrm{~cm})$ & 17,0 & 30,7 & 42,2 & 55,5 & 67,3 & 76,8 & 85,5 & 93,1 & 100,0 & $16.518,4$ \\
\hline $4(0-10 \mathrm{~cm})$ & 19,2 & 34,8 & 49,1 & 63,9 & 76,3 & 85,7 & 92,1 & 96,5 & 100,0 & $3.263,8$ \\
\hline $4(20-40 \mathrm{~cm})$ & 15,9 & 25,3 & 37,2 & 55,0 & 70,3 & 82,7 & 91,4 & 96,6 & 100,0 & $6.370,6$ \\
\hline $5(0-10 \mathrm{~cm})$ & 11,7 & 23,7 & 35,9 & 49,4 & 67,8 & 89,1 & 96,3 & 98,8 & 100,0 & $18.577,4$ \\
\hline $5(20-40 \mathrm{~cm})$ & 10,3 & 21,8 & 33,2 & 55,9 & 73,0 & 84,4 & 92,1 & 96,7 & 100,0 & $13.726,7$ \\
\hline $6(0-10 \mathrm{~cm})$ & 29,7 & 42,5 & 54,0 & 64,8 & 71,5 & 78,0 & 83,8 & 90,5 & 100,0 & 724,6 \\
\hline $6(20-40 \mathrm{~cm})$ & 10,9 & 43,4 & 65,6 & 90,4 & 91,4 & 91,4 & 96,9 & 99,3 & 100,0 & 34,7 \\
\hline $7(0-10 \mathrm{~cm})$ & 21,4 & 35,0 & 45,9 & 59,5 & 71,7 & 81,5 & 89,2 & 95,1 & 100,0 & $3.226,8$ \\
\hline $7(20-40 \mathrm{~cm})$ & 18,4 & 31,1 & 43,2 & 58,1 & 70,5 & 80,8 & 88,7 & 94,8 & 100,0 & $1.589,3$ \\
\hline $8(0-10 \mathrm{~cm})$ & 25,7 & 40,2 & 51,6 & 67,8 & 78,7 & 87,7 & 93,0 & 96,9 & 100,0 & $8.731,1$ \\
\hline $8(20-40 \mathrm{~cm})$ & 25,6 & 45,9 & 59,9 & 72,9 & 81,7 & 89,7 & 94,4 & 97,6 & 100,0 & $3.435,8$ \\
\hline
\end{tabular}

(1) Agrupamento dos solos de acordo com os ambientes de deposição de Pb provenientes da mineração 1: referência, sem deposição de $\mathrm{Pb} ; 2,4$ e 5: incorporação de resíduos da metalurgia ao perfil; 3, 6 e 8: adição de Pb particulado via chaminés da fábrica; 8: contaminação por passagem da água pluvial pela fábrica, que escorre em direção ao rio Ribeira. ${ }^{(2)}$ (Teor dessorvido no tempo t/teor total dessorvido no tempo de $1.382 \mathrm{~h}$ ) $\times 100$. Por exemplo, no tempo acumulado de $14 \mathrm{~h}$ (primeira extração de $2 \mathrm{~h}+$ segunda de $12 \mathrm{~h}$ ), dividiu-se o teor acumulado das duas primeiras extrações pelo teor dessorvido acumulado em $1.382 \mathrm{~h}$.

inversão desse comportamento no solo 4 (maiores teores acumulados na camada de 20 a $40 \mathrm{~cm}$ - Quadro 3) foi atribuída à maior contaminação direta na subsuperfície, em razão da grande mistura de rejeitos da metalurgia no perfil.

A equação de difusão parabólica descreveu satisfatoriamente a cinética de liberação do $\mathrm{Pb}$ dos solos com ácido cítrico (Quadro 4). O padrão de duas fases de liberação (duas retas com declividades distintas, com altos coeficientes de ajustes (valores de $\mathrm{R}^{2}$ ) e baixos valores de Erro Padrão Estimado - quadro 4 e figuras 2, 3, 4 e 5) também foi observado por outros autores em estudos de dessorção de $\mathrm{Pb}$ (Elkhatib et al., 1992; Strawn \& Sparks, 2000). Em todos os solos foi observada liberação mais rápida na primeira fase, ou seja, o valor de $k$ (coeficiente angular ou declividade da reta) foi maior (Quadro 4).

A maior liberação na primeira fase deve estar relacionada à liberação de $\mathrm{Pb}$ adsorvido em sítios de menor energia de adsorção (esfera externa). Com exceção do solo 6, as amostras apresentaram elevados valores de CTC e ocorrência de minerais 2:1 secundários (Quadro 2), o que favorece a adsorção não específica de metais pesados (Fontes et al., 2001). Já na segunda fase as reações de dessorção envolveriam reações de troca de ligantes $\left(\mathrm{H}^{+}\right.$do ácido cítrico por $\mathrm{Pb}^{2+}$ ligado covalentemente) e, ou, dissolução de grupos funcionais de superfície e liberação de $\mathrm{Pb}$ (ou de $\left.\mathrm{Pb}(\mathrm{OH})^{+}\right)$adsorvidos por complexo de esfera interna nos grupos funcionais da matéria orgânica e nos grupos aluminol (-AlOH) e ferrol (-FeOH) nas bordas dos óxidos de Fe e Al e da caulinita (Stevenson, 1971; Kalbasi et al., 1978; Canellas et al., 1999; Araújo \& Amaral Sobrinho, 2000; Coles \& Yong, 2002; MirandaTrevino \& Coles, 2003; Yavuz et al., 2003).

Os solos 2, 4 e 5 (Figura 2), com visível presença de rejeitos da metalurgia nos perfis (observações de campo - Quadro 1), apresentaram altos teores de $\mathrm{Pb}$ dessorvidos acumulados em $1.382 \mathrm{~h}$ de cinética (Quadro 3). A textura arenosa da amostra de 20 a $40 \mathrm{~cm}$ do solo 5 (Quadro 2) deveu-se à grande ocorrência de rejeitos grosseiros do tamanho areia, o que pode ter contribuído para menor solubilização de $\mathrm{Pb}$ nos três primeiros tempos de extração em relação aos solos 2 e 4 . O coeficiente angular da reta na segunda fase da cinética das amostras desse ponto também foi inferior (Quadro 4). A contaminação no solo 2 não foi por destinação direta dos rejeitos sobre o solo, mas sua posição intermediária na encosta do terreno favoreceu o acúmulo de colúvio (mistura de sedimentos e rejeitos da metalurgia) vindo das cotas mais altas. O maior coeficiente angular da equação da reta ajustada ao solo 2 na profundidade de 20 a $40 \mathrm{~cm}$ (5,37 na primeira fase - Quadro 4) em relação ao solo 5 refletiu o efeito favorável à solubilização do rejeito por sua mistura íntima aos coloides do solo no processo erosivo. No ponto 5 observou-se que os rejeitos da mineração ainda estavam preservados e com maior ocorrência na superfície do solo. Já no solo 2, no estudo do perfil, verificou-se que as partículas de rejeitos 
Quadro 4. Coeficientes da equação parabólica de difusão $\left(M t / M o=a+k t^{1 / 2}\right)$, coeficientes de ajustes $\left(R^{2}\right)$ e Erros Padrões Estimados (EPE) para duas fases de liberação de Pb em amostras de solo coletadas em diferentes pontos de antiga área de mineração, no município de Adrianópolis-PR

\begin{tabular}{|c|c|c|c|c|c|}
\hline \multirow[b]{2}{*}{ Amostra } & \multirow[b]{2}{*}{ Fase } & \multicolumn{2}{|c|}{ Coeficientes } & \multirow[b]{2}{*}{$\mathbf{R}^{2}$} & \multirow[b]{2}{*}{$\begin{array}{c}\text { EPE (valores } \\
\left.\text { multiplicados por } 10^{4}\right)^{(1)}\end{array}$} \\
\hline & & $\begin{array}{c}\text { Linear (a) (valores } \\
\text { multiplicados por } 10^{2} \text { ) }\end{array}$ & $\begin{array}{c}\text { Angular (k) (valores } \\
\text { multiplicados por } 10^{2} \text { ) }\end{array}$ & & \\
\hline $1(0-10 \mathrm{~cm})$ & $\frac{1}{2}$ & $\begin{array}{r}2,52 \\
54,02\end{array}$ & $\begin{array}{l}3,81 \\
1,24\end{array}$ & 0,9896 & $\begin{array}{r}15,80 \\
0,37\end{array}$ \\
\hline $1(20-40 \mathrm{~cm})$ & $\frac{1}{2}$ & $\begin{array}{l}72,10 \\
93,28\end{array}$ & $\begin{array}{l}1,17 \\
0,19\end{array}$ & 0,9199 & $\begin{array}{r}11,75 \\
0,22\end{array}$ \\
\hline $2(0-10 \mathrm{~cm})$ & $\frac{1}{2}$ & $\begin{array}{l}13,81 \\
67,61\end{array}$ & $\begin{array}{l}3,77 \\
0,90\end{array}$ & 0,9889 & $\begin{array}{r}13,13 \\
1,23\end{array}$ \\
\hline $2(20-40 \mathrm{~cm})$ & $\frac{1}{2}$ & $\begin{array}{l}10,23 \\
59,06\end{array}$ & $\begin{array}{l}5,37 \\
1,18\end{array}$ & 0,9594 & $\begin{array}{l}0,87 \\
8,03\end{array}$ \\
\hline $3(0-10 \mathrm{~cm})$ & $\frac{1}{2}$ & $\begin{array}{l}17,36 \\
67,25\end{array}$ & $\begin{array}{l}3,62 \\
0,89\end{array}$ & 0,9855 & $\begin{array}{r}15,91 \\
0,93\end{array}$ \\
\hline $3(20-40 \mathrm{~cm})$ & $\frac{1}{2}$ & $\begin{array}{l}11,31 \\
51,60\end{array}$ & $\begin{array}{l}4,86 \\
1,37\end{array}$ & 0,9759 & $\begin{array}{l}1,17 \\
5,69\end{array}$ \\
\hline $4(0-10 \mathrm{~cm})$ & $\begin{array}{l}1 \\
2\end{array}$ & $\begin{array}{l}16,41 \\
74,42\end{array}$ & $\begin{array}{l}4,73 \\
0,72\end{array}$ & 0,9901 & $\begin{array}{r}52,78 \\
1,98\end{array}$ \\
\hline $4(20-40 \mathrm{~cm})$ & $\frac{1}{2}$ & $\begin{array}{l}11,63 \\
78,85\end{array}$ & $\begin{array}{l}4,15 \\
0,58\end{array}$ & 0,9934 & $\begin{array}{l}8,53 \\
0,72\end{array}$ \\
\hline $5(0-10 \mathrm{~cm})$ & $\frac{1}{2}$ & $\begin{array}{r}6,34 \\
91,04\end{array}$ & $\begin{array}{l}4,60 \\
0,25\end{array}$ & 0,9996 & $\begin{array}{l}0,56 \\
0,23\end{array}$ \\
\hline $5(20-40 \mathrm{~cm})$ & $\frac{1}{2}$ & $\begin{array}{r}6,02 \\
80,59\end{array}$ & $\begin{array}{l}4,65 \\
0,53\end{array}$ & 0,9886 & $\begin{array}{r}17,38 \\
0,53\end{array}$ \\
\hline $6(0-10 \mathrm{~cm})$ & $\frac{1}{2}$ & $\begin{array}{l}22,76 \\
54,49\end{array}$ & $\begin{array}{l}5,11 \\
1,25\end{array}$ & 0,9988 & $\begin{array}{r}0,194 \\
0,73\end{array}$ \\
\hline $6(20-40 \mathrm{~cm})$ & $\frac{1}{2}$ & $\begin{array}{r}-3,41 \\
86,28\end{array}$ & $\begin{array}{r}11,49 \\
0,40\end{array}$ & 0,9957 & $\begin{array}{l}7,24 \\
1,83\end{array}$ \\
\hline $7(0-10 \mathrm{~cm})$ & $\frac{1}{2}$ & $\begin{array}{l}15,71 \\
59,41\end{array}$ & $\begin{array}{l}4,80 \\
1,17\end{array}$ & 0,9601 & $\begin{array}{l}0,94 \\
7,57\end{array}$ \\
\hline $7(20-40 \mathrm{~cm})$ & $\begin{array}{l}1 \\
2\end{array}$ & $\begin{array}{l}17,69 \\
72,04\end{array}$ & $\begin{array}{l}3,76 \\
0,76\end{array}$ & 0,9872 & $\begin{array}{r}14,21 \\
0,64\end{array}$ \\
\hline $8(0-10 \mathrm{~cm})$ & $\frac{1}{2}$ & $\begin{array}{l}22,72 \\
77,75\end{array}$ & $\begin{array}{l}4,41 \\
0,62\end{array}$ & 0,9911 & $\begin{array}{r}8,12 \\
12,25\end{array}$ \\
\hline $8(20-40 \mathrm{~cm})$ & $\stackrel{1}{2}$ & $\begin{array}{l}20,56 \\
75,11\end{array}$ & $\begin{array}{l}5,93 \\
0,73\end{array}$ & 0,9880 & $\begin{array}{l}8,91 \\
5,56\end{array}$ \\
\hline
\end{tabular}

(1) Erro Padrão Estimado $=\left[\Sigma\left(\mathrm{M}_{\mathrm{t}}-\mathrm{M}_{\mathrm{t}}{ }^{*}\right)^{2}\right] /(\mathrm{n}-2)$, em que Mt e Mt* representam os teores de $\mathrm{Pb}$ obtidos nas extrações e calculados nas equações de cinética (valores observados e estimados), respectivamente, para o tempo t, e n é o número de pontos determinados (Simard et al., 1992).
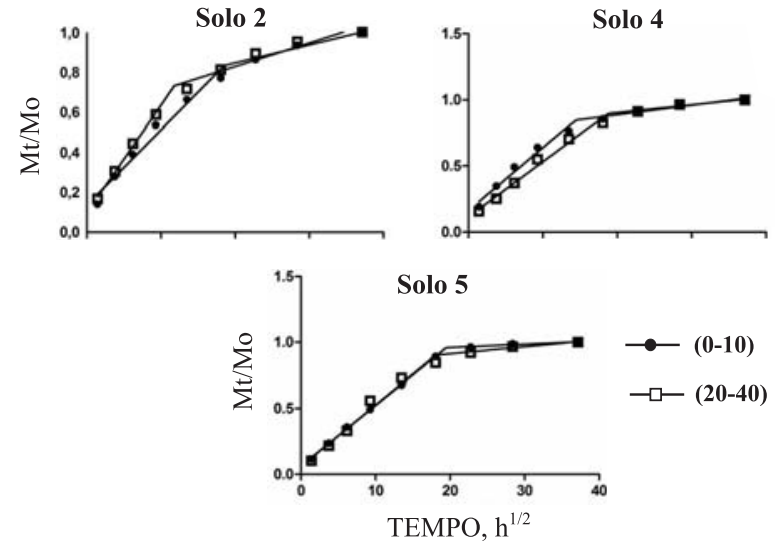

Figura 2. Cinética de liberação de $\mathrm{Pb}$ [equação parabólica de difusão $\left.\left(\mathrm{Mt} / \mathrm{Mo}=\mathbf{a}+\mathrm{kt}^{1 / 2}\right)\right]$ das amostras de solos contaminadas com rejeito de metalurgia, em área localizada no município de Adrianópolis-PR.

estavam distribuídas ao longo dos horizontes mais superficiais (depositados via colúvio - evidência morfológica de descontinuidade litológica) e em estado mais avançado de alteração (menor tamanho e perda do aspecto vítreo). As amostras coletadas nas duas profundidades do solo 2 apresentaram comportamentos semelhantes quanto ao teor dessorvido acumulado de $\mathrm{Pb}$ (cerca de $3.500 \mathrm{mg} \mathrm{kg}^{-1}$ - Quadro 3).

Os solos contaminados sob influência de material particulado emitido pelas chaminés da antiga fábrica foram representados pelos solos 3, 6 e 7 (Figura 3). Outros estudos na mesma área também identificaram que a deposição atmosférica foi a fonte responsável pela intensa contaminação de $\mathrm{Pb}$ nos solos argilosos do entorno da fábrica (Andrade et al., 2009a,b; Barros et al., 2010a,b). Considera-se que a contaminação oriunda de material particulado proporcionou maior risco ao meio ambiente e à população, pois, segundo Andrade et al (2009b), essa fonte de contaminação determinou os maiores teores de $\mathrm{Pb}$ biodisponível na área. O maior teor dessorvido em $1.382 \mathrm{~h}$ no solo 3 (Quadro 3) refletiu sua maior proximidade a uma das chaminés da fábrica e deveu-se às suas características favoráveis ao acúmulo de $\mathrm{Pb}$ (altos teores de matéria orgânica e argila e ocorrência de esmectita - Quadro 2); o somatório desses dois efeitos (deposição e acúmulo) resultou em maior contaminação do solo. As amostras de solo do ponto 3 (local mais próximo à fábrica - 

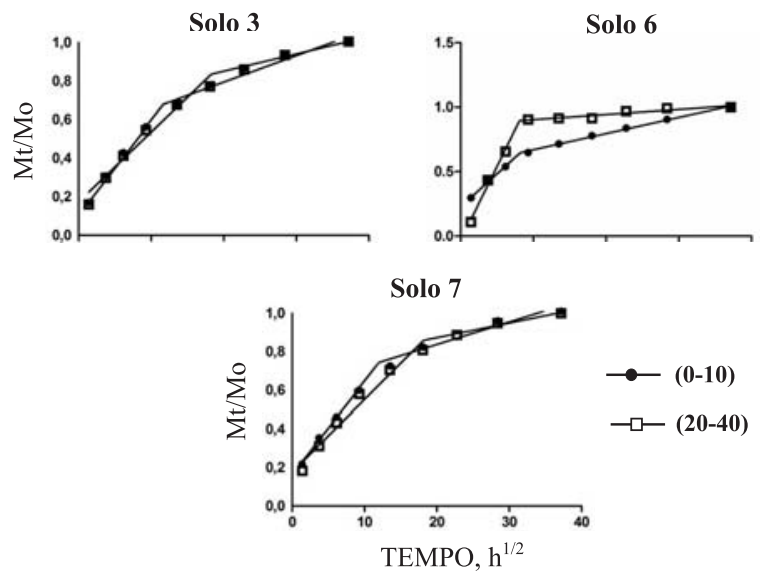

Figura 3. Cinética de liberação de $\mathrm{Pb}$ [equação parabólica de difusão $\left.\left(\mathrm{Mt} / \mathrm{Mo}=\mathbf{a}+\mathrm{kt}^{1 / 2}\right)\right] \mathrm{das}$ amostras de solos contaminadas com material particulado oriundo das chaminés da fábrica, em área localizada no município de Adrianópolis-PR.

Quadro 1) foram coletadas às margens de uma estrada na saída do pátio da fábrica e no caminhamento da fumaça de uma das chaminés. O solo 6 , um pouco mais distante da fábrica (Quadro 1), estava localizado no antigo trajeto da fumaça expelida pela chaminé principal. Por ser formado a partir do intemperismo de quartzito (Quadro 1), sua textura arenosa (Quadro 2) não favoreceu a retenção e acúmulo de $\mathrm{Pb}$ no perfil (Quadro 3). Os teores dessorvidos acumulados em $1.382 \mathrm{~h}$ foram relativamente baixos comparados aos das demais amostras, nas duas profundidades (724,6 e 34,7 $\left.\mathrm{mg} \mathrm{kg}^{-1}\right)$, superando apenas os teores da amostra de referência 1 . Aliado aos baixos teores de argila, o solo 6 apresentou os menores teores de carbono orgânico. Quanto à cinética de liberação, verificou-se que na profundidade de 20 a $40 \mathrm{~cm}$ do solo 6 o $\mathrm{Pb}$ foi liberado mais rapidamente (maior coeficiente angular em relação às demais amostras - Quadro 4), refletindo a pouca retenção devido aos baixos teores de argila e matéria orgânica do solo. A maior distância vertical e horizontal do solo 7 à fabrica (Quadro 1) reduziu a sua contaminação com $\mathrm{Pb}$ particulado, em relação ao solo 3 (Quadro 3).

$\mathrm{O}$ ambiente contaminado por influência de lavagem da fábrica (rica em poeiras e resíduos da metalurgia) por água pluvial (faixa compreendida entre o rio Ribeira e a fábrica - Figura 1) foi representado pelo ponto 8 (Quadros 3 e 4 e Figura 4). Em avaliação preliminar da área (dados não publicados), os teores totais de $\mathrm{Pb}$ nas pilhas de resíduos de vários aspectos e cores deixadas no pátio da fábrica chegaram à ordem de $200.000 \mathrm{mg} \mathrm{kg}^{-1}$. A porção mais superficial do solo 8, devido à constante recarga de $\mathrm{Pb}$ com a água de escorrimento superficial e ao menor potencial de adsorção de metal, por sua menor CTC e teor de argila (Quadro 2), apresentou maior liberação acumulada de $\mathrm{Pb}$ em 1.382 h $\left(8.731,1 \mathrm{mg} \mathrm{kg}{ }^{-1}\right)$, em relação à camada de 20 a $40 \mathrm{~cm}\left(3.435,8 \mathrm{mg} \mathrm{kg}^{-1}\right.$ de solo).

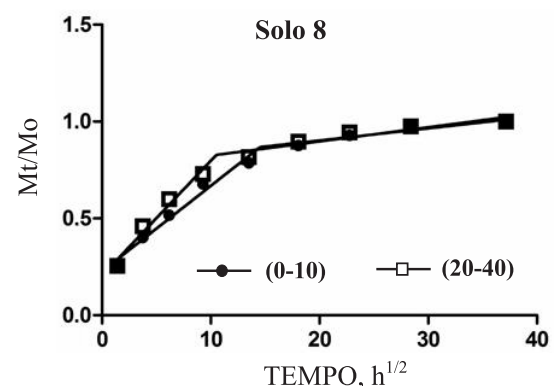

Figura 4. Cinética de liberação de $\mathrm{Pb}$ [equação parabólica de difusão $\left.\left(\mathrm{Mt} / \mathrm{Mo}=\mathrm{a}+\mathrm{kt}^{1 / 2}\right)\right]$ das amostras de solo contaminadas pela passagem das águas pluviais de lavagem da fábrica, em área localizada no município de AdrianópolisPR.

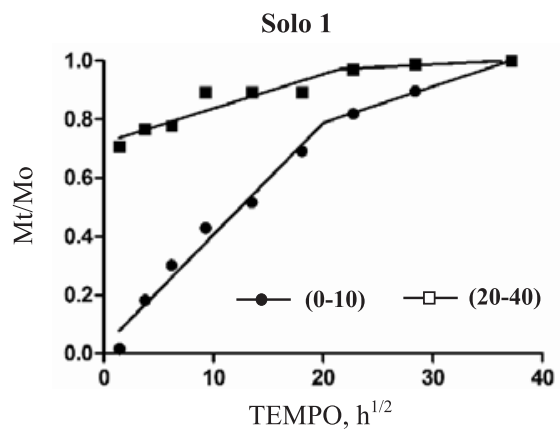

Figura 5. Cinética de liberação de $\mathrm{Pb}$ [equação parabólica de difusão $\left.\left(\mathrm{Mt} / \mathrm{Mo}=\mathrm{a}+\mathrm{kt}^{1 / 2}\right)\right] \mathrm{da}$ amostra de referência dos teores naturais de metal, em área localizada no município de Adrianópolis-PR.

\section{CONCLUSÕES}

1. A descrição da cinética de liberação de $\mathrm{Pb}$ pela equação parabólica de difusão mostrou que o processo ocorreu, com diferentes taxas, em duas fases, sendo a taxa de liberação maior na primeira fase.

2. A amostra de subsuperfície do solo 6 (arenoso) apresentou a maior velocidade (taxa) de dessorção de $\mathrm{Pb}$. Contudo, o reduzido acúmulo do metal durante as atividades de mineração e metalurgia na área (teor total de apenas $48,7 \mathrm{mg} \mathrm{kg}^{-1}$ ) sugere baixo risco ambiental deste solo.

3. Mesmo apresentando baixo teor de argila (48 $\mathrm{g} \mathrm{kg}^{-1}$ ), a alta ocorrência de resíduos da metalurgia do tamanho areia na amostra de 0 a $10 \mathrm{~cm}$ do solo 5 resultou em elevado tamponamento de $\mathrm{Pb}$, com teor dessorvido acumulado do metal em $1.382 \mathrm{~h}$ de $18.577,4 \mathrm{mg} \mathrm{kg}^{-1}$.

4. O solo 3 argiloso mais próximo da antiga fábrica e sujeito à adição de $\mathrm{Pb}$ particulado oriundo da chaminé também foi considerado crítico para contaminação ambiental, com elevada liberação já na primeira extração (2 h de contato: $2.808,1 \mathrm{mg} \mathrm{kg}^{-1}$ ). 
5. A incorporação dos rejeitos da metalurgia no perfil favoreceu sua solubilização e disponibilidade de $\mathrm{Pb}$.

\section{LITERATURA CITADA}

ALI, I.A.; KAFKAFI, U.; YAMAGUCHI, I.; SUGIMOTO, Y. \& INANAGA, S. Growth, transpiration, root-born, cytokinins and gibberelins, and nutrient compositional changes in seasame exposed to low root zone temperature under different ratios of nitrate. J. Plant Nutr., 23:123$140,2000$.

ALLOWAY, B.J. Heavy metals in soils. New York, John Wiley, 1990. 339p.

AMARAL SOBRINHO, N.M.B.; VELLOSO, A.C.X.; COSTA, L.M. \& OLIVEIRA, C. Mobilidade de metais pesados em solo tratado com resíduo siderúrgico. R. Bras. Ci. Solo, 22:345-353, 1998.

ANDRADE, M. Teores de metais pesados em águas e em sedimentos de área de mineração e processamento de $\mathrm{Pb}$, no município de Adrianópolis-PR. Curitiba, Universidade Federal do Paraná, 2009. 114p. (Tese de Mestrado)

ANDRADE, M.G.; MELO, V.F.; GABARDO, J.; SOUZA, L.C.P. \& REISSMANN, C.B. Metais pesados em solos de área de mineração e metalurgia de chumbo: I - Fitoextração. R. Bras. Ci. Solo, 33:1879-1888, 2009a.

ANDRADE, M.G.; MELO, V.F.; SOUZA, L.C.P.; GABARDO, J. \& REISSMANN, C.B. Metais pesados em solos de área de mineração e metalurgia de chumbo: II - formas e disponibilidade. R. Bras. Ci. Solo, 33:1889-1897, 2009 b.

ARAÚJO, W.S. \& AMARAL SOBRINHO, N.M.B. Influência das propriedades físicas e químicas de solos intemperizados na adsorção de chumbo, cobre e zinco. Flor. Amb., 7:167$180,2000$.

BARROS, Y.J.; MELO, V.F.; DIONÍSIO, J.A.; CARON, L.; OLIVEIRA, E.B.; AZEVEDO, J.C.R. \& SOUZA, L.C.P. Indicadores de qualidade de solos de área de mineração e metalurgia de chumbo: I- microbiologia. R. Bras. Ci. Solo, 34: 2010a. (aceito - no prelo) ??VERIFICAR??

BARROS, Y.J.; MELO, V.F.; SAUTTER, K.D.; BUSCHLE, B.; OLIVEIRA, E.B.; AZEVEDO, J.C.R. \& SOUZA, L.C.P. Indicadores de qualidade de solos de área de mineração e metalurgia de chumbo: II - mesofauna e plantas. R. Bras. Ci. Solo, 34: 2010b. (aceito - no prelo) - ??VERIFICAR??

BOSSO, S.T.; ENZWEILER, J. \& ANGÉLICA, R. Lead bioacessibility in soil and mine wastes after immobilization with phosphates. Water Air Soil Pollut., 195:257-273, 2008.

CANELLAS, L.P.; SANTOS, G.A.; AMARAL SOBRINHO, N.M.B.; MORAES, A. \& RUMAJAMEK, V.M. Adsorção de $\mathrm{Cu}^{+2}$ e $\mathrm{Cd}^{+2}$ em ácidos húmicos extraídos de resíduos orgânicos de origem urbana. Ci. Rural, 29:21-26, 1999.

CASSIANO, A.M. Fonte de contaminação por elementos-traço na bacia do rio Ribeira de Iguape (SP-PR): Estratégia para a remediação de uma área de disposição de rejeitos de mineração de Pb-Ag. São Carlos, Universidade de São Paulo, 2001. 159p. (Tese de Doutorado)
CASTILHOS, R.M.V. \& MEURER, E.J. Cinética de liberação de potássio em Planossolo do Estado do Rio Grande do Sul. Ci. Rural, 31:1-10, 2001.

CHLOPECKA, A.; BACON, J.R.; WILSON, M.J. \& KAY, J. Forms of cadmium, lead and zinc in contaminated soils from southwest Poland. J. Environ. Quality, 25: 69-79, 1996.

COLES, C.A. \& YONG, R.N. Aspects of kaolinite characterization and retention of $\mathrm{Pb}$ and $\mathrm{Cd}$. Applied Clay Sci., 22:39-45, 2002.

DIAS-JÚNIOR, H.E.; MOREIRA, F.M.S.; SIQUEIRA, J.O. \& SILVA, R. Metais pesados, densidade e atividade microbiana em solo contaminado por rejeitos de indústria de zinco. R. Bras. Ci. Solo, 22:631-640, 1998.

ELKHATiB, E.A.; ElSheBINY, G.M. \& BALBA, A.M. Comparison of four equations to describe the kinetics of lead desorption from soils. J. Plant Nutr. Soil Sci., 155:285291, 1992.

EYSINK, G.G.J. Metais pesados no Vale do Ribeira e em Iguape-Cananéia. Ambiente, 2:6-13, 1988.

FONTES, M.P.F.; CAMARGO, O.A. \& SPOSITO, G. Eletroquímica das partículas coloidais e sua relação com a mineralogia de solos altamente intemperizados. Sci. Agric., 18:265-271, 2001.

HUNDAL L.S. \& PASRICHA, N.S. Nonexchangeable potassium release kinetics in illitic soil profiles. Soil Sci., 156:34-41, 1993.

HUNDAL L.S. \& PASRICHA, N.S. Adsorption-desorption kinetics of potassium as influenced by temperature and background anions. Geoderma, 83:215-225, 1998.

KABATA-PENDIAS, A. \& PENDIAS, H. Trace elements in soils and plants. 3.ed. Boca Raton, CRC, 2001. 413p.

KALBASI, M.; RACZ, G.J. \& RUDGERS, L.A. Mechanism of zinc adsorption by iron and aluminum oxides. Soil Sci., 125:146-150, 1978.

KUMMER, L. Mineralogia e fracionamento de metais pesados em solos de área de mineração e processamento de chumbo, Adrianópolis (PR). Curitiba, Universidade Federal do Paraná, 2008. 140p. (Tese de Mestrado)

LIM, C.H. \& JACKSON, M.L. Dissolution for total elemental analysis. In: PAGE, A.L. ed. Methods of soil analysis. Madison, American Society of Agronomy, 1986. p.1-12.

McGRATH, S.P. \& ZHAO, F.J. Phytoextraction of metals and metalloids from contaminated soils. Current Opinion Biotechnol., 14:277-282, 2003.

MELO, V.F.; CORREAA, G.F.; RIBEIRO, A.N. \& MASCHIO, P.A. Cinética de liberação de potássio e chumbo pelos minerais da fração argila de solos do triângulo mineiro. R. Bras. Ci. Solo, 29:533-545, 2005.

MEURER, E.J. \& CASTILHOS, R.M.V. Liberação de potássio de frações de solos e sua cinética. R. Bras. Ci. Solo, 25:823$829,2001$.

MIRANDA-TREVINO, J.C. \& COLES, C.A. Kaolinite properties, structure and influence of metal retention on pH. Applied Clay Sci., 23:133-139, 2003. 
ROCA, J. \& POMARES, F. Prediction of available heavy metals by six chemical extractants in a sewage sludge-amended soil. Comm. Soil Sci. Plant Anal., 22:2119-2136, 1991.

SIMARD, R.R.; De KIMPE, C.R. \& ZIZKA, J. Release of potassium and magnesium from soil fractions and its kinetics. Soil Sci. Soc. Am. J., 56:1421-1428, 1992.

SONG, S.K \& HUANG, P.M. Dynamics of potassium release from potassium-bearing minerals as influenced by oxalic and citric acids. Soil Sci. Soc. Am. J., 52:383-390, 1988.

STEVENSON, F.J. Nature of divalent transition metal complexes of humic acids as revealed by a modified potentiometric titration method. Soil Sci., 123:10-17, 1977.
STRAWN, D.G. \& SPARKS, D.L. Effects of soil organic matter on the kinetics and mechanisms of $\mathrm{Pb}(\mathrm{II})$ : Sorption and desorption in soil. Soil Sci. Soc. Am. J, 64:144-156, 2000.

XU, X.Y.; MCGRATH, S.P. \& ZHAO, F.J. Rapid reduction of arsenate in the medium mediated by plant roots. New Phytol., 176:590-599, 2007.

WANG, W.S.; SHAN, X.Q.; WEN, B. \& ZHANG, S.Z. Relationship between the extractable metals from soils and metals taken up by maize roots and shoots. Chemosphere, 53:523-530, 2003.

YAVUZ, O.; ALTUNKAYNAK, Y. \& GÜZEL, F. Removal of copper, nickel, cobalt and manganese from aqueous solution by kaolinite. Water Res., 37:948-952, 2003. 